Please do not remove this page

RMIT

UNIVERSITY

\title{
Effects of plasma-treatment on the electrical and optoelectronic properties of layered black phosphorus
}

Kuriakose, Sruthi; Ahmed, Taimur; Balendhran, Shiva; Collis, Gavin; Bansal, Vipul; Aharonovich, Igor; Sriram, Sharath

https://researchrepository.rmit.edu.au/esploro/outputs/9921861714301341/filesAndLinks?institution=61RMIT_INST\&index=null

Kuriakose, S., Ahmed, T., Balendhran, S., Collis, G., Bansal, V., Aharonovich, I., Sriram, S., Bhaskaran, M., \& Walia, S. (2018). Effects of plasma-treatment on the electrical and optoelectronic properties of layered black phosphorus. Applied Materials Today, 12, 244-249. https://doi.org/10.1016/j.apmt.2018.06.001 Document Version: Accepted Manuscript

Published Version: https://doi.org/10.1016/j.apmt.2018.06.001

Repository homepage: https://researchrepository.rmit.edu.au (C) 2018 Elsevier Ltd. All rights reserved.

Downloaded On 2023/04/26 20:56:45 +1000 
Thank you for downloading this document from the RMIT Research Repository.

The RMIT Research Repository is an open access database showcasing the research outputs of RMIT University researchers.

RMIT Research Repository: http://researchbank.rmit.edu.au/

\section{Citation:}

Kuriakose, S, Ahmed, T, Balendhran, S, Collis, G, Bansal, V, Aharonovich, I, Sriram, S, Bhaskaran, M and Walia, S 2018, 'Effects of plasma-treatment on the electrical and optoelectronic properties of layered black phosphorus', Applied Materials Today, vol. 12, pp. 244-249.

See this record in the RMIT Research Repository at:

https://researchbank.rmit.edu.au/view/rmit:47942

Version: Accepted Manuscript

\section{Copyright Statement:}

(C) 2018. This manuscript version is made available under the CC-BY-NC-ND 4.0 license http://creativecommons.org/licenses/by-nc-nd/4.0/

\section{Link to Published Version:}

https://doi.org/10.1016/j.apmt.2018.06.001 


\section{Effects of plasma-treatment on the electrical and optoelectronic properties of layered black phosphorus}

Sruthi Kuriakose, Taimur Ahmed, Sivacarendran Balendhran, Gavin E. Collis, Vipul Bansal, Igor Aharonovich, Sharath Sriram, Madhu Bhaskaran and Sumeet Walia*

Ms. S. Kuriakose, Mr. T. Ahmed, Dr. S. Balendhran, Assoc. Prof. S. Sriram, Assoc. Prof. M. Bhaskaran, Dr. S. Walia

Functional Materials and Microsystems Research Group and Micro Nano Research Facility, RMIT University, Melbourne VIC 3001, Australia

*E-mail: sumeet.walia@rmit.edu.au

Dr. G.E. Collis

CSIRO Manufacturing, CSIRO, Bayview Avenue, Clayton 3168, Victoria, Australia

Prof. V. Bansal

Ian Potter NanoBioSensing Facility and NanoBiotechnology Research Laboratory, School of Science, RMIT University, Melbourne VIC 3001, Australia

Assoc. Prof. I. Aharonovich

School of Mathematical and Physical Sciences, University of Technology Sydney, Ultimo, NSW 2007, Australia

KEYWORDS: phosphorene, black phosphorus, plasma thinning, etching, photoresponse 


\begin{abstract}
Exfoliated few-layer black-phosphorus (BP) has been explored for a variety of electrical and optoelectronic applications. Plasma-assisted thinning of BP has emerged as an exciting pathway to achieve BP crystals of desired thickness. However, to fully realise the true potential of plasma-assisted thinning of $\mathrm{BP}$ and other emerging 2D materials, it is critical to understand the effects of different plasma environments on the electrical and optoelectronic properties of the resultant material. Here, we investigate the influence of $\mathrm{Ar}$ and $\mathrm{O}_{2}$ plasma on the electrical and optoelectronic properties of plasma-treated BP flakes. It is revealed that by manipulating the environment under which $\mathrm{BP}$ is exposed to the plasma, it is possible to engineer defects that lead to new photoluminescence (PL) emission peaks without compromising the switching ratios or carrier mobilities of BP-based field effect transistors (FETs). Overall, our study finds the use of $\mathrm{O}_{2}$ plasma as a more suitable approach to retain and enrich the intrinsic (opto)electronic properties of BP. Additionally, our study, for the first time, experimentally reveals the ability of BP to respond to UV excitation.
\end{abstract}

\title{
Introduction
}

Since the discovery of graphene, two-dimensional materials (2D) have received remarkable attention, in particular, due to their exceptional transport properties.[1] The weak inter layer and strong intra layer forces in the bulk form of these materials have enabled the realization of pristine, planar materials via seamless processes such as exfoliation. The fascinating properties that arise in planar materials due to the quantum confinement of carriers, makes them attractive for deployment as functional materials in transistors,[2-6] photodetectors,[712] solar cells,[9,13] batteries,[14,15] energy storage systems,[9,16,17] catalysis,[18] and sensing[15,19-21] to name a few. While graphene has been extensively investigated, a major limitation associated with its close to zero inherent band gap has subsequently sparked a 
search for other 2D materials with natural band gaps. This led to the advent of transition metal dichalcogenides (TMDCs) such as $\mathrm{MoS}_{2}$ that has direct band gap in its monolayer form but carrier mobilities that are inferior to that of graphene.[22,23]

More recently, elemental analogues have emerged as an exciting class of materials.[24] Amongst them, black phosphorus (BP) and its monolayer form commonly referred as 'phosphorene' have highly desirable intrinsic characteristics, with one of them being its direct bandgap that is independent of thickness but its value is tunable depending on the number of layers $(0.2 \mathrm{eV}$ in bulk to $1.5 \mathrm{eV}$ in monolayer $\mathrm{BP})$.[1,12] Furthermore, it possesses high carrier mobilities of the order of $10^{3} \mathrm{~cm}^{2} / \mathrm{V} . \mathrm{s},[1]$ with theoretical predictions indicating that it can be as high as $20,000 \mathrm{~cm}^{2} /$ V.s.[25] Additionally, its highly anisotropy-dependant thermal, electrical, and mechanical properties[26,27] are in striking contrast to other 2D TMDCs and graphene.

So far, the most common method of realising layered crystals of BP is mechanical exfoliation. The layers obtained are typically of different thicknesses which can be identified under the optical microscope and using atomic force microscopy (AFM). Generally, the highest proportion of flakes obtained using mechanical exfoliation are in the thickness range of $15-35 \mathrm{~nm}$, with a scattered few that are less than $10 \mathrm{~nm}$ thick. This introduces a high degree of randomness in the thicknesses achieved. For several applications such as field effect transistors (FETs), a higher proportion of thinner layers are vital to fully capitalise on the quantum confinement effects.[22] Furthermore, even being the most stable allotrope of phosphorus,[28] BP is not environmentally stable and undergoes rapid photo-oxidation in ambient conditions, which is further expedited in the presence of humidity,[29-35] leading to surface defects and carrier scattering centres that compromise the promising electrical characteristics of this 2D material. 
Plasma-assisted thinning of mechanically-exfoliated BP flakes has recently emerged as a promising route to control the number of layers in a BP crystal on-demand.[22,36,37] Two common gases involved in this approach include either argon (Ar) or oxygen $\left(\mathrm{O}_{2}\right)$; however their relative pros and cons are not investigated. To fully realise the true potential of plasmaassisted thinning of $\mathrm{BP}$ and other emerging $2 \mathrm{D}$ materials, it is critical to understand the effects of different plasma environments on BP thinning, its ambient stability and importantly, on the electrical and optoelectronic properties of the resultant material.

The current study focuses on these important aspects of plasma-assisted BP thinning, and, as such, offers new insights into the influence of $\mathrm{Ar}$ and $\mathrm{O}_{2}$ plasma on the electrical and optoelectronic properties of plasma-treated BP flakes. Our investigations reveal that by manipulating the environment under which BP is exposed to the plasma, it is possible to engineer defects that lead to new photoluminescence (PL) emission peaks without compromising the switching ratios or carrier mobilities of BP-based field effect transistors (FETs). Overall, our study finds the use of $\mathrm{O}_{2}$ plasma as a more suitable approach to retain and enrich the intrinsic (opto)electronic properties of BP. Additionally, our study, for the first time, experimentally reveals the ability of BP to respond to UV excitation.

\section{Experimental Section}

Sample preparation and storage: The BP flakes are mechanically exfoliated from a bulk crystal (Smart Elements) on to plasma-cleaned $300 \mathrm{~nm} \mathrm{SiO} / 2$ Si substrates. The BP surface gets photo-oxidised in ambient air, so the samples were prepared and stored in a nitrogen atmosphere. BP flakes of uniform thicknesses of $25 \mathrm{~nm}$ to $30 \mathrm{~nm}$ were identified via optical contrast. Photo lithography followed by electron beam evaporation of metal electrodes (Au 
$90 \mathrm{~nm}$ with adhesion layer of $\mathrm{Cr} 10 \mathrm{~nm}$ ), was carried out to realise two-terminal devices based on BP flakes.

\section{Characterization:}

AFM analyses are performed on a Veeco Dimension Icon in ScanAsyst mode. Plasma etching is performed with the PlasmaPro System100 Inductive Coupled Plasma (Oxford Instruments) system at room temperature. $\mathrm{O}_{2}$ plasma is generated at ICP power of $400 \mathrm{~W}$ and $\mathrm{RF}$ power of $30 \mathrm{~W}$ with $\mathrm{O}_{2}$ flow rate of 30 s.c.c.m. at a pressure of $10 \mathrm{mTorr}$. Ar etching is carried out at ICP power of $300 \mathrm{~W}$ and RF power at $30 \mathrm{~W}$ with Ar flow of 15 s.c.c.m at a pressure of 5 mTorr. The etch rates calculated for $\mathrm{O}_{2}$ and Ar plasma are $0.12 \mathrm{~nm} / \mathrm{s}$ and $0.50 \mathrm{~nm} / \mathrm{s}$, respectively. The Raman spectroscopy and photoluminisence characterizations were performed using a Horiba LabRAM HR Evolution Micro-Raman system equipped with a $532 \mathrm{~nm}$ laser source (50× objective). Photoexcitation was carried out using a $280 \mathrm{~nm}$ wavelength, high power excitation source (Thorlabs, Inc). The incident power at the sample surface is measured using the commercial UV power meter (Newport Corporation). All photoresponse experiments are performed in dark conditions in a customised environmental chamber (Linkam) at room temperature. The electrical measurements are performed using the Keithley 4200SCS semiconductor parameter analyser.

\section{Results and Discussion}

Few-layer BP is mechanically-exfoliated from a bulk crystal (Smart Elements) using a blue Nitto tape on to plasma-cleaned Si substrates with a $300 \mathrm{~nm}$ thermally-grown $\mathrm{SiO}_{2}$ layer. We will refer to these as-exfoliated flakes as 'pristine BP' henceforth in the manuscript. The exfoliation process is carried out in UV deficient dark conditions and the samples are stored in an inert nitrogen $\left(\mathrm{N}_{2}\right)$ atmosphere to preserve the integrity of the samples during the exfoliation process (see Experimental for details).[29,38] For plasma treatment, BP flakes of 
thicknesses in the range of 20-25 nm are initially identified using optical contrast with respect to the underlying $\mathrm{SiO}_{2} / \mathrm{Si}$ substrate.

The thickness of the pristine BP flakes is confirmed using atomic force microscopy (AFM) (Figure 1a,b). To establish an optimal plasma etching recipe that provides controlled layer removal of BP without inducing significant damages on the morphology and crystal structure, various radio frequency (RF) and inductively-coupled plasma (ICP) power conditions at fixed pressure of 10 mTorr are initially investigated. After a series of etch cycles, each followed by AFM characterization, an optimal set of RF and ICP powers for both Ar and $\mathrm{O}_{2}$ etching (see Experimental for details) are established, given their different ionization efficiencies. At these powers, no significant optical changes to the surface are observed following the etching process. The selected pristine BP flakes (as shown in Figures 1a,b) are plasma-treated in Ar and $\mathrm{O}_{2}$ atmospheres individually. Both pristine BP flakes are etched down to sub-10 nm thicknesses (Figure 1c,d) with controlled exposure. We noted that the process can be implemented to successfully achieve monolayers however, we observed that thinner layers are prone to rapid degradation and further plasma-treatment will not allow for a valid comparison, especially in the case of Ar etched BP, where there is no process-generated $\mathrm{P}_{x} \mathrm{O}_{y}$ protective coating. [36] Further, it is known that a thinner BP channel is more susceptible to potential variations in the substrate due to the formation of traps at the interface. The carrier mobility is known to increase monotonically in the thickness range $4-12 \mathrm{~nm}$ due to charge screening effects. However, further increases in channel thickness result in higher interlayer resistance of $\mathrm{BP}$, thereby reducing the carrier conduction from the electrical contacts formed on the top surfaces of the BP.[37] It is also noted from the AFM characterisation that there is an increase in average surface roughness from $0.611 \mathrm{~nm}$ and $0.727 \mathrm{~nm}$ for the pristine BP flakes to $1.310 \mathrm{~nm}$ and $1.510 \mathrm{~nm}$ for the $\mathrm{Ar}$ and $\mathrm{O}_{2}$ etched flakes, respectively. This surface roughness is comparable to the value reported previously.[37] The etch rate is calculated to 
be $0.50 \mathrm{~nm} / \mathrm{s}$ for Ar and $0.12 \mathrm{~nm} / \mathrm{s}$ for $\mathrm{O}_{2}$ plasma thinning, showing a faster etch rate for $\mathrm{Ar}$ plasma assisted thinning.

Figure 2 illustrates a schematic representation of the plasma bombardment and the BP layer removal process. In the case of $\mathrm{O}_{2}$ plasma treatment, it has been reported[36] that a layer of $\mathrm{P}_{x} \mathrm{O}_{y}$ forms on the $\mathrm{BP}$ surface which covers and protects the underlying layers of $\mathrm{BP}$ as shown in Figure 2. This is evident from a 4-times lower $\mathrm{O}_{2}$ plasma layer-removal rate of $\mathrm{BP}$ in comparison to that of Ar plasma assisted thinning. Once an equilibrium is reached between the formation and etching of the surface $\mathrm{P}_{x} \mathrm{O}_{y}$ layer, this slower etching process allows a highly controllable thinning of the underlying BP.[36] On the other hand, while Ar plasma removes any potentially pre-existing ambient oxide layer, the inability of Ar plasma to induce $\mathrm{P}_{x} \mathrm{O}_{y}$ layer formation directly causes a more rapid layer-by-layer etching of BP atoms, which also manifests as a higher etching rate compared to $\mathrm{O}_{2}$ plasma.[22,37]

To determine the effects of the plasma treatment process on the compositional integrity of BP, we conducted micro-Raman spectroscopic analysis on the same BP flakes before and after the etching processes. A comparison of the Raman spectra shows the presence of all three signature peaks of $\mathrm{BP}$ corresponding to $\mathrm{A}_{\mathrm{g}}^{1}\left(361 \mathrm{~cm}^{-1}\right), \mathrm{B}_{2 \mathrm{~g}}\left(438 \mathrm{~cm}^{-1}\right)$ and the $\mathrm{A}_{\mathrm{g}}^{2}$ $\left(465 \mathrm{~cm}^{-1}\right)$ modes (Figure 3a,b). ${ }^{[39]}$ The $\mathrm{A}_{\mathrm{g}}^{1}$ mode originates from the out-of-plane vibrations of phosphorus atoms along the $c$-axis, while the $\mathrm{B}_{2 \mathrm{~g}}$ and $\mathrm{A}_{\mathrm{g}}^{2}$ modes arise from the in-plane vibrations of phosphorus atoms along the $b$-axis (armchair) and $a$-axis (zigzag), respectively. It is known that the integrated Raman intensity ratio of $\mathrm{A}_{\mathrm{g}}^{2}$ to $\mathrm{A}_{\mathrm{g}}^{1}$ increases monotonically with decreasing layer numbers, and therefore, can be used as a reliable fingerprint to determine the thickness trend. In our case, the intensity ratios of $\mathrm{A}_{\mathrm{g}}^{2} / \mathrm{A}_{\mathrm{g}}^{1}$ increases after plasma-assisted etching which indicates reduction in the thicknesses.[22,39] However, this ratio can be significantly influenced by surface oxidation and therefore AFM remains the best tool to directly measure the BP thickness. 
To further investigate the effects of plasma treatment in different environments, we compare the photoluminescence (PL) spectra of the pristine and etched BP flakes (Figure 3c). Typically, only mono/bilayers of BP show PL in the visible region.[22] However, as seen from Figure 3c, the $\mathrm{O}_{2}$ plasma treated flake reveals a prominent peak between 650 and $760 \mathrm{~nm}$. There are no PL signals observed from the Ar plasma treated BP, which is also of a similar thickness as the $\mathrm{O}_{2}$ etched BP. The emergence of this PL signature can be attributed to the presence of in-gap defect states. During the $\mathrm{O}_{2}$ plasma etching, the oxygen ions create neutral and electrically metastable defects in BP.[36,40] These defects are expected to introduce an acceptor state near the conduction band, and consequently, a disturbed valence band state is formed by the hybridisation of the $p$-orbitals of $\mathrm{O}$ and $\mathrm{P}$ atoms.[40,41] These defects are energetically confined to one of the defect bands within the band gap of the material.[40] They can become electrically active when exposed to activation energies larger than their energy state. To verify this hypothesis, we conducted a transient photoresponse study on pristine, Ar and $\mathrm{O}_{2}$ plasma etched BP flakes. Furthermore, the introduction of defects during the $\mathrm{O} 2$ plasma etching process enables oxidation and the dissociation of oxygen dimers thereby facilitating the formation of a $\mathrm{P}_{x} \mathrm{O}_{y}$ layer at the top surface.[42]

The photoresponse is acquired using a $280 \mathrm{~nm}$ excitation source. The low wavelength source will provide energy larger than the defect and the natural energy states and therefore induce a noticeable response. For this purpose, two terminal devices are fabricated (see Experimental) and the BP channel current is measured upon pulsed UV excitation. The measurements are carried out under constant channel bias of $100 \mathrm{mV}$. The illumination covers the whole active device area at a constant incident power of $1 \mathrm{~mW} / \mathrm{cm}^{2}$. Figure 4a,b shows the behaviour of the channel current under the exposure to $280 \mathrm{~nm}$ for two pristine BP flakes. It is seen that the current reversibly increases upon exposure. This is expected due to the photogeneration of carriers in the direct bandgap material such as BP. Furthermore, it should be noted that in the 
case of elemental materials such as 2D BP, the interstitial $\mathrm{P}$ atom forms two bonds with other $\mathrm{P}$ atoms in adjacent layers and leaves one dangling bond.[40] Hence, the formation energies of the point defects are generally lower than other 2D systems, resulting in the creation of spin-polarized p-type states, which are predicted to be the origin of p-type conductivity. This also implies that such defects do not act as sinks for majority carriers (holes), due to the lack of compensating electrons and therefore an increase in current is seen upon photoexcitation with a $280 \mathrm{~nm}$ source.[40,43]

Following Ar plasma treatment, there is a distinctive change in the photoresponse trend. The channel current decreases upon illumination as seen from Figure 4c, in contrast to what is observed in the pristine BP flake before etching (Figure 4a). This intriguing behaviour is repeatedly observed in multiple devices following Ar etching. We explain this based on the thickness-dependent creation of reactive oxygen species (ROS), the formation of which results in a drop of electrical conductivity.[33] Thinner BP is more susceptible to the formation of oxide traps as a result of the interplay between light and environmental oxygen.[44-46] In every cycle of $280 \mathrm{~nm}$ exposure post-etching with Ar plasma, a decrease in conductivity is observed which is in contrast to the pristine BP device (Figure 4a and c). In the case of a pristine device, the energy gap of the thicker BP is relatively small $(\sim 0.2 \mathrm{eV})$, which makes the ROS formation due to photo-oxidation much slower and hence an expected increase in electrical conductivity is seen upon photo-excitation. However, when the BP is significantly thinned down and is not capped with any protective layer (as is the case after $\mathrm{Ar}$ etching), the larger energy gap allows an efficient formation of ROS that are known to degrade the electrical conductivity of BP.[29,42] This explanation is further supported with a model established by Martel and co-workers[47] which suggests that the rate of oxidation depends linearly on the oxygen concentration and light intensity and exponentially on the square of the energy gap. 
On the other hand, following $\mathrm{O}_{2}$ plasma treatment, the photoresponse in ambient conditions is observed to increase which is similar in trend to that of the pre-treated pristine BP flake (Figure 4d). However, the magnitude of response is relatively lower after etching. This can be attributed to the formation of an encapsulating surface $\mathrm{P}_{x} \mathrm{O}_{y}$ layer,[36] which typically limits the incident photon energy. In contrast to the Ar-etched BP, there is no change in the photoresponse trend. This can be attributed to the protective nature of the $\mathrm{P}_{x} \mathrm{O}_{y}$ layer that inhibits an efficient formation of ROS that could have otherwise degraded the electrical characteristics.

To evaluate the effect of the plasma process on the transport properties of BP, field effect transistors (FETs) are fabricated (see Experimental for details) and subjected to plasma etching. The transfer characteristics are acquired for two BP flakes pre- and post-treatment with $\mathrm{Ar}$ and $\mathrm{O}_{2}$ plasma, as shown in Figure $\mathbf{5}$ (see Supplementary Information Figure S2 for the corresponding $I-V$ curves). Optical micrographs of the devices pre- and post-treatment, are shown as insets. The thicknesses of the pristine BP flakes are 25-30 nm. The etched $\mathrm{Ar}$ and $\mathrm{O}_{2}$ FET device have BP layers with thickness of $\sim 9 \mathrm{~nm}$ and $5 \mathrm{~nm}$, respectively. The switching ratio $\left(I_{\mathrm{ON}} / I_{\mathrm{OFF}}\right)$ increases from 8 and 3 for the pristine BP-based FETs to approximately $2 \times 10^{3}$ for the Ar and $1 \times 10^{4}$ for the $\mathrm{O}_{2}$ etched BP FETs, respectively. This is a dramatic increase of at least three orders of magnitude in both cases. Such an increase is in line with the previously reported values for FETs based on similar thicknesses of BP.[3,7,48] We also calculate the carrier mobilities using the following equation:[49]

$$
\mu=\frac{\Delta I_{\mathrm{ds}}}{\Delta V_{\mathrm{ds}}} \times \frac{L}{\text { W.C. } V_{\mathrm{ds}}}
$$

where $\frac{\Delta I_{\mathrm{DS}}}{\Delta V_{\mathrm{DS}}}$ is the transconductance, $L$ is the channel length, $W$ is the channel width, $C$ is the gate oxide capacitance (115 $\mu \mathrm{Fm}^{-1}$, see Supplementary Information, Figure S2 for this calculation) and the drain-source voltage $\left(V_{\mathrm{DS}}\right)$ is $50 \mathrm{mV}$. The carrier mobility for the $\mathrm{Ar}$ and 
$\mathrm{O}_{2}$ etched BP are found to be $336 \mathrm{~cm}^{2} / \mathrm{V} \cdot \mathrm{s}$ and $411 \mathrm{~cm}^{2} / \mathrm{V} \cdot \mathrm{s}$, respectively. These values are in agreement with those reported for as exfoliated BP crystals of similar thickness at room temperature.[3,7,43,48] Based on these results, it can be concluded that the plasma process $\left(\mathrm{Ar}\right.$ and $\mathrm{O}_{2}$ ) has not resulted in any discernible change in the expected electronic properties of BP.[50]

In our study, we verify observations from previous studies that both $\mathrm{Ar}$ and $\mathrm{O}_{2}$ plasmatreatment processes are viable and effective in controlling layer thickness. However, we reveal that $\mathrm{O}_{2}$ plasma etching provides distinct advantages. Firstly, it offers an opportunity to controllably introduce defects that give rise to PL emission in the near infrared region, without compromising its electrical properties as demonstrated by fabricating a FET and a photosensor. Further, it results in the formation of a surface protective $\mathrm{P}_{x} \mathrm{O}_{y}$ encapsulation layer thereby offering a better thickness control due to a slower etch rate. Based on known facts deduced from other reports[36,51] we also note that the formation of an encapsulation layer in the case of $\mathrm{O}_{2}$ etching also offers higher ambient stability compared to the Ar-etched BP where the absence of a native encapsulation layer can in fact speed up the process of degradation and may require additional steps to protect the thinned BP flakes.

\section{Conclusions}

In summary, we have demonstrated the effects of plasma-assisted layer control of BP in Ar and $\mathrm{O}_{2}$ atmospheres on the electrical and optoelectronic properties. Using spectroscopy, photoconductivity, and FET characteristics, it is shown that the $\mathrm{O}_{2}$ plasma etching is favourable as it offers superior thickness control, primarily due to the formation of a surface $\mathrm{P}_{x} \mathrm{O}_{y}$ encapsulation layer and the resultant defects can be exploited to generate PL emissions at new wavelengths while simultaneously retaining its electrical performance. A comparison of the structure and fundamental electronic characteristics before and after etching reveals 
that both $\mathrm{Ar}$ and $\mathrm{O}_{2}$ assisted thinning preserve the intrinsic properties of $2 \mathrm{D} \mathrm{BP}$. It is also shown that pristine BP could potentially be implemented as the functional layer for UV sensors, wherein the direction of resistance change can be manipulated using the etching process. Although a relatively slow response time may be beneficial in exploiting the UV response for applications in optically controlled memory devices.

\section{Acknowledgements}

S.K. and T.A. contributed equally. The authors acknowledge support from the Australian Research Council (ARC) for personnel and project support via DE150100909 (S.B.), FT140101285 (V.B.), DP170103477 (V.B.), DE160100023 (M.B.), DP140100170 (M.B.), and equipment funding through LE0882246, LE0989615, and LE150100001.

\section{References}

1. K. Cho, et al., "Phosphorene: An emerging 2D material," J. Mater. Sci, 32, 2017pp. 28392847,

2. H. Liu, et al., "Phosphorene: an unexplored 2D semiconductor with a high hole mobility," ACS Nano, 8, 2014.p. 4033,

3. L. Li, et al., "Black phosphorus field-effect transistors," Nat. Nanotechnol, 9, 2014.p. 372,

4. $\quad$ S. F. Chowdhury, et al., "Black Phosphorous Thin-Film Transistor and RF Circuit Applications," IEEE Electron Device Lett., 37, 2016.p. 449,

5. Y. Y. Illarionov, et al., "Bias-temperature instability in single-layer graphene field-effect transistors," Appl. Phys. Lett., 105, 2014.p. 143507,

6. J. O. Island, et al., "Environmental instability of few-layer black phosphorus," 2D Mater, 2, 2015.p. 011002,

7. F. Xia, et al., "Rediscovering black phosphorus as an anisotropic layered material for optoelectronics and electronics," Nat. Commun., 5, 2014.p. 4458,

8. M. Engel, et al., "Black phosphorus photodetector for multispectral, high-resolution imaging," Nano Lett., 14, 2014.p. 6414,

9. M. Akhtar, et al., "Recent advances in synthesis, properties, and applications of phosphorene," npj 2D Mater. Appl., 1, 2017.p. 5,

10. M. Singh, et al., "Soft Exfoliation of 2D SnO with size-dependent optical properties," 2D Mater, 4, 2017.p. 025110,

11. P. Sahatiya, et al., "2D MoS 2-carbon quantum dot hybrid based large area, flexible UV-visNIR photodetector on paper substrate," Appl Mater Today., 10, 2018pp. 106-114,

12. D. Li, et al., "Black phosphorus polycarbonate polymer composite for pulsed fibre lasers," Appl Mater Today., 4, 2016pp. 17-23,

13. W. Hu, et al., "Edge-modified phosphorene nanoflake heterojunctions as highly efficient solar cells," Nano Lett., 16, 2016.p. 1675,

14. C. M. Park, et al., "Black phosphorus and its composite for lithium rechargeable batteries," Adv Mater, 19, 2007.p. 2465,

15. V. Eswaraiah, et al., "Black phosphorus nanosheets: synthesis, characterization and applications," Small, 12, 2016pp. 3480-3502,

16. W. Li, et al., "Ultrafast and directional diffusion of lithium in phosphorene for highperformance lithium-ion battery," Nano Lett., 15, 2015pp. 1691-1697, 
17. K. Kalantar-zadeh, et al., "Two dimensional and layered transition metal oxides," Appl Mater Today., 5, 2016pp. 73-89,

18. H. Wang, et al., "Ultrathin black phosphorus nanosheets for efficient singlet oxygen generation," J. Am. Chem. Soc, 137, 2015.p. 11376,

19. P. Li, et al., "Air-stable black phosphorus devices for ion sensing," ACS Appl Mater Inter, 7, 2015pp. 24396-24402,

20. S. Cui, et al., "Ultrahigh sensitivity and layer-dependent sensing performance of phosphorene-based gas sensors," Nat Comm, 6, 2015.p. 8632,

21. L. Kou, et al., "Phosphorene as a superior gas sensor: selective adsorption and distinct I-V response," J. Phys. Chem. Lett., 5, 2014pp. 2675-2681,

22. W. Lu, et al., "Plasma-assisted fabrication of monolayer phosphorene and its Raman characterization," Nano Res, 7, 2014.p. 853,

23. Y. Liu, et al., "Layer-by-layer thinning of MoS2 by plasma," ACS Nano, 7, 2013.p. 4202,

24. S. Balendhran, et al., "Elemental analogues of graphene: silicene, germanene, stanene, and phosphorene," Small, 11, 2015pp. 640-652,

25. A. Castellanos-Gomez, et al., "Isolation and characterization of few-layer black phosphorus," 2 D Mater, 1, 2014.p. 025001,

26. G. Abellán, et al., "Fundamental Insights into the Degradation and Stabilization of Thin Layer Black Phosphorus," J. Am. Chem. Soc, 139, 2017.p. 10432,

27. J. Tao, et al., "Mechanical and electrical anisotropy of few-layer black phosphorus," ACS Nano, 9, 2015.p. 11362,

28. A. J. Mannix, et al., "Synthesis and chemistry of elemental 2D materials," Nat Rev Chem, 1, 2017.p. 0014,

29. T. Ahmed, et al., "Degradation of black phosphorus is contingent on UV-blue light exposure," npj 2D Mater. Appl., 1, 2017.p. 18,

30. S. Walia, et al., "Ambient Protection of Few-Layer Black Phosphorus via Sequestration of Reactive Oxygen Species," Adv Mater, 29, 2017.p. 1700152,

31. S. Walia, et al., "Defining the role of humidity in the ambient degradation of few-layer black phosphorus," 2D Mater., 4, 2016.p. 015025,

32. S. C. Dhanabalan, et al., "Emerging Trends in Phosphorene Fabrication towards Next Generation Devices," Adv Sci, 4, 2017.p. 1600305,

33. Q. Zhou, et al., "Light-Induced Ambient Degradation of Few-Layer Black Phosphorus: Mechanism and Protection," Angew. Chem. Int. Ed., 128, 2016pp. 11609-11613,

34. G. Wang, et al., "Phosphorene oxide: stability and electronic properties of a novel twodimensional material," Nanoscale, 7, 2015pp. 524-531,

35. S. Kuriakose, et al., "Black phosphorus: Ambient degradation and strategies for protection," 2D Mater, 5, 2018

36. J. Pei, et al., "Producing air-stable monolayers of phosphorene and their defect engineering," Nat. Commun., 7, 2016.p. 10450,

37. J. Jia, et al., "Plasma-treated thickness-controlled two-dimensional black phosphorus and its electronic transport properties," ACS Nano, 9, 2015.p. 8729,

38. J. Qiao, et al., "High-mobility transport anisotropy and linear dichroism in few-layer black phosphorus," Nat Comm, 5, 2014.p. 4475,

39. A. Łapińska, et al., "Temperature Evolution of Phonon Properties in Few-Layer Black Phosphorus," J. Phys. Chem. C, 120, 2016pp. 5265-5270,

40. A. Ziletti, et al., "Oxygen defects in phosphorene," Phys Rev Lett, 114, 2015.p. 046801,

41. Y. Y. Illarionov, et al., "Highly-stable black phosphorus field-effect transistors with low density of oxide traps," npj 2D Mater. Appl., 1, 2017.p. 23,

42. K. L. Utt, et al., "Intrinsic defects, fluctuations of the local shape, and the photo-oxidation of black phosphorus," ACS Cent Sci., 1, 2015pp. 320-327, 
43. A. Carvalho, et al., "Phosphorene: from theory to applications," Nat Rev Mater, 1, 2016.p. 16061,

44. Y. Liu, et al., "Two-dimensional mono-elemental semiconductor with electronically inactive defects: the case of phosphorus," Nano Lett., 14, 2014.p. 6782,

45. J. Li, et al., "Structure and electronic properties of grain boundaries in earth-abundant photovoltaic absorber Cu2ZnSnSe4," ACS Nano, 5, 2011.p. 8613,

46. W. J. Yin, et al., "Engineering Grain Boundaries in Cu2ZnSnSe4 for Better Cell Performance: A First-Principle Study," Adv Energy Mater, 4, 2014.p. 1300712,

47. A. Favron, et al., "Photooxidation and quantum confinement effects in exfoliated black phosphorus," Nat Mater, 14, 2015pp. 826-832,

48. M. Buscema, et al., "Fast and broadband photoresponse of few-layer black phosphorus fieldeffect transistors," Nano Lett., 14, 2014.p. 3347,

49. S. Balendhran, et al., "Enhanced charge carrier mobility in two-dimensional high dielectric molybdenum oxide," Adv Mater, 25, 2013.p. 109,

50. S. Liu, et al., "Thickness-dependent Raman spectra, transport properties and infrared photoresponse of few-layer black phosphorus," J. Mater. Chem. C, 3, 2015.p. 10974,

51. M. Edmonds, et al., "Creating a stable oxide at the surface of black phosphorus," ACS Appl Mater Inter, 7, 2015pp. 14557-14562,
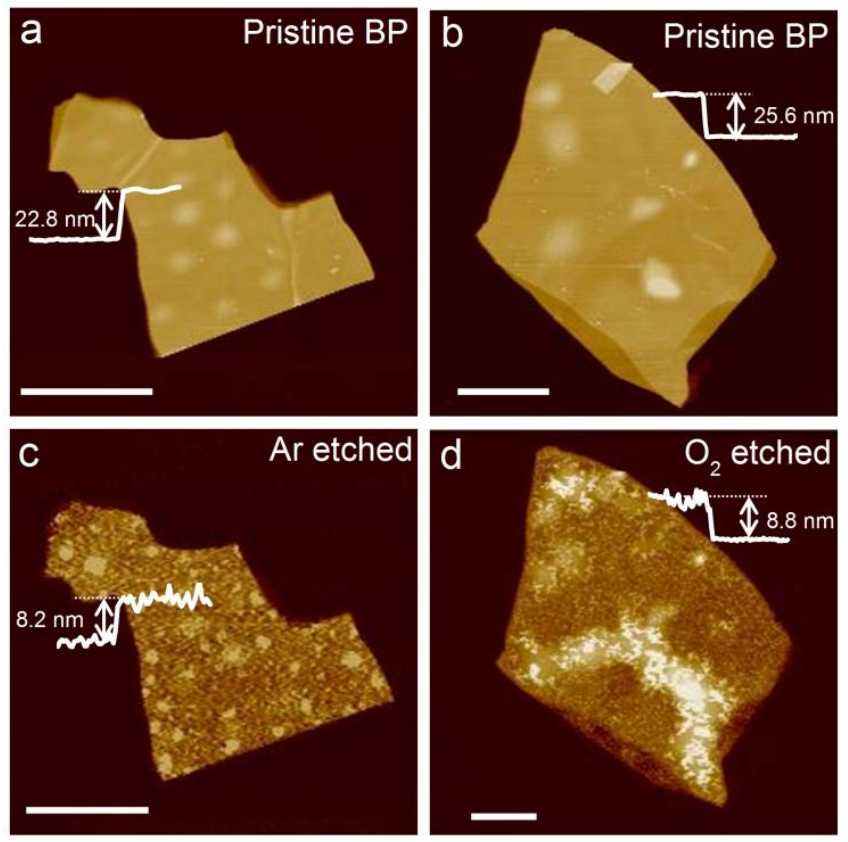

Figure 1: The AFM images of the pristine BP flakes (a), (b) and after plasma-assisted etching with $\mathrm{Ar}(\mathrm{c})$ and $\mathrm{O}_{2}$ plasma (d) respectively along with their corresponding thickness profiles. The scale bars represent $2 \mu \mathrm{m}$. 


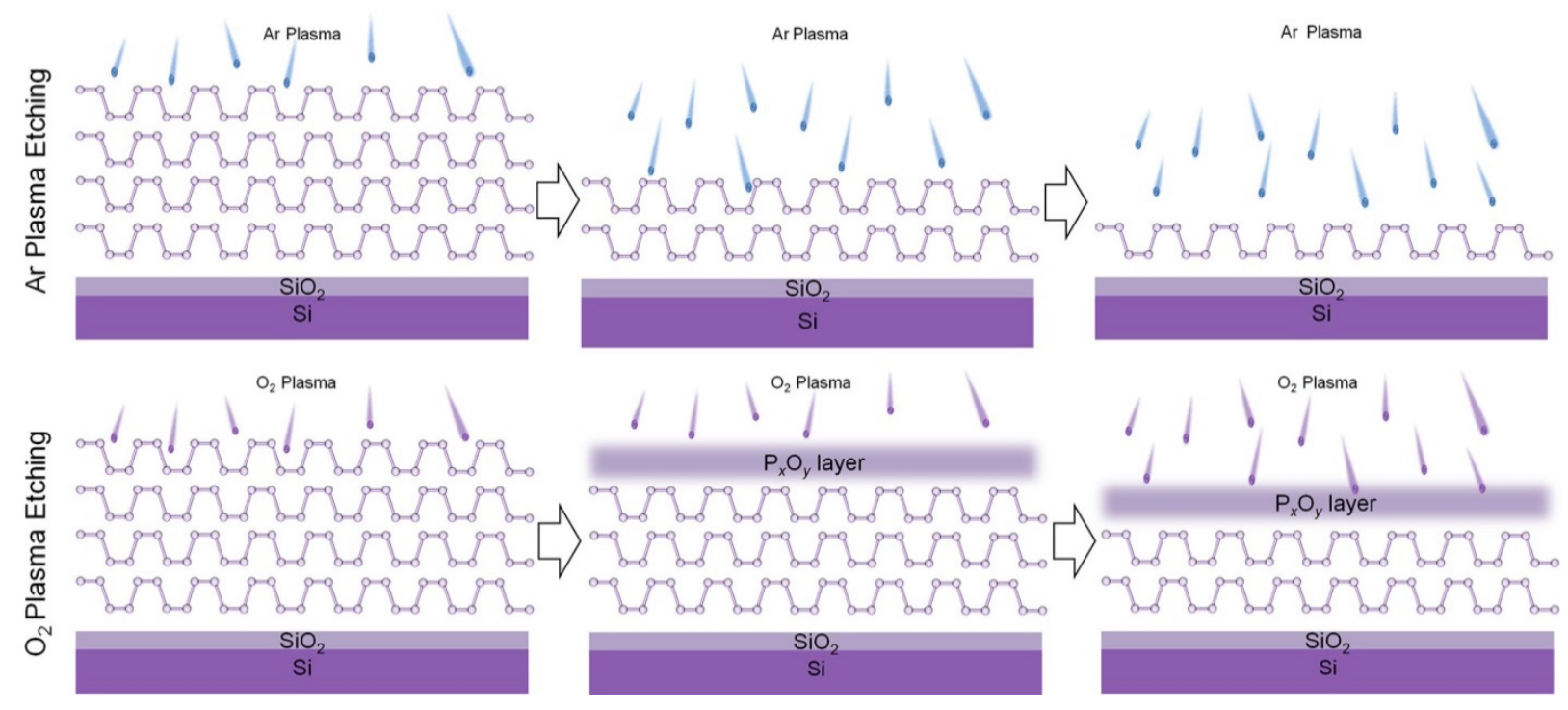

Figure 2: A schematic representation of the plasma interaction with the BP. The $\mathrm{O}_{2}$ plasma forms a $\mathrm{P}_{x} \mathrm{O}_{y}$ layer which acts as a shield for the underlying $\mathrm{BP}$, giving more control for the etching process compared to that of the Ar plasma. The Ar etching process results in a systematic layer-by-layer removal of any $\mathrm{P}_{x} \mathrm{O}_{y} / \mathrm{BP}$ layers.
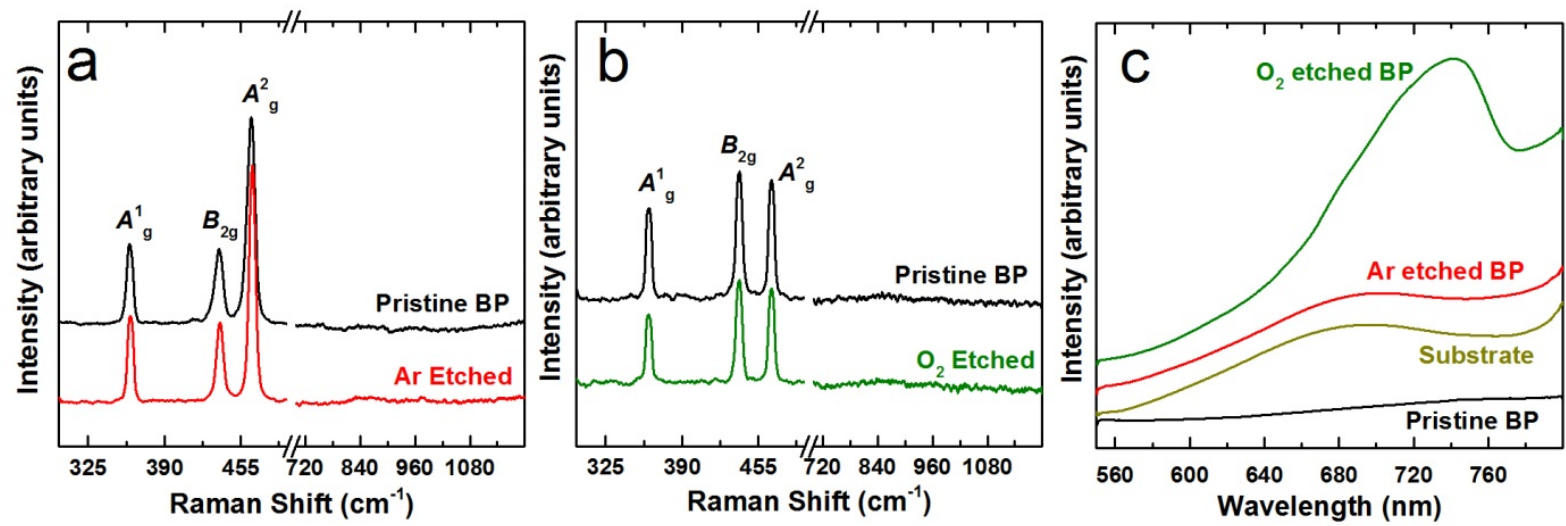

Figure 3: A comparison of the Raman spectra for the pristine and (a) Ar-etched and (b) $\mathrm{O}_{2-}$ etched BP. (c) Photoluminescence spectra comparison of the Ar- and $\mathrm{O}_{2}$-etched BP flakes on a $\mathrm{SiO}_{2} / \mathrm{Si}$ substrate. 

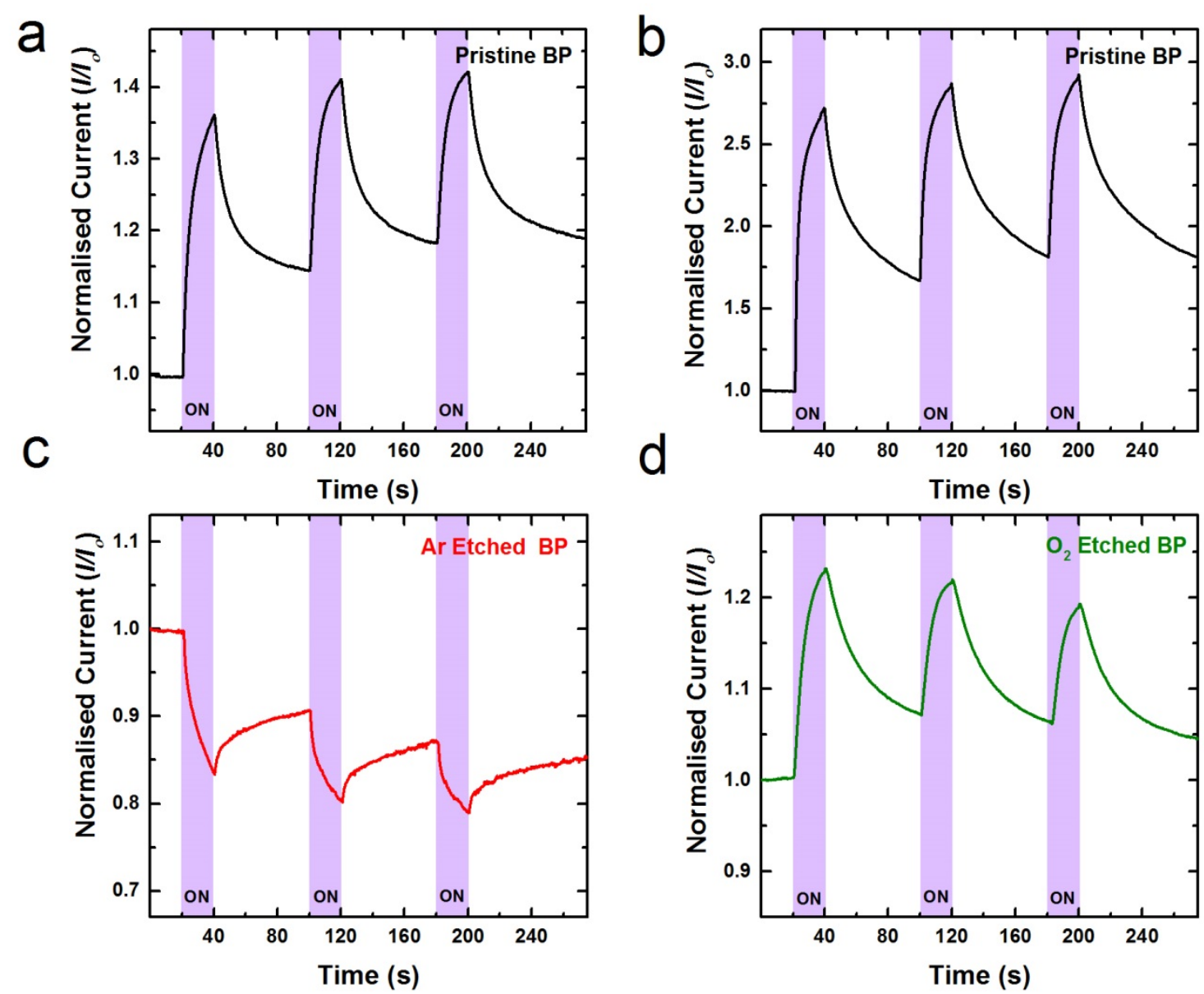

Figure 4: Photoresponse comparison of the BP flakes in air under pulsed illumination of $280 \mathrm{~nm}$ wavelength with a channel bias of $100 \mathrm{mV}$. The normalised current is plotted against time for two different devices based on pristine BP flakes (a and b) and with Ar-etched (c) and $\mathrm{O}_{2}$-etched (d) BP flakes in. The ON (shaded areas) indicate that the $280 \mathrm{~nm}$ source is illuminating the device. The measurements are performed with cycles of $20 \mathrm{~s}$ exposure followed by 60 s recovery under dark conditions. 
a

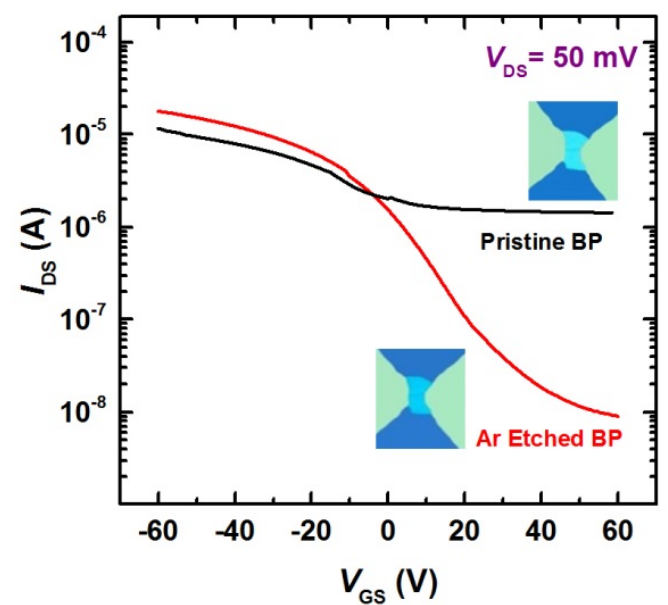

b

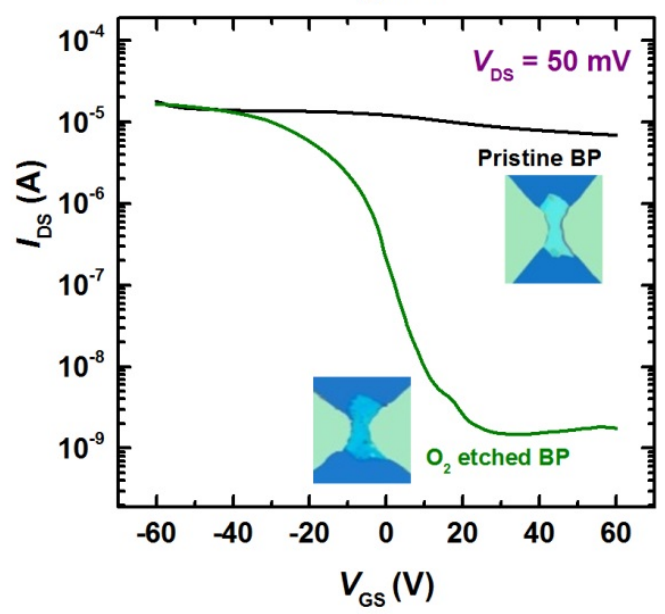

Figure 5: Transconductance comparison of (a) pristine and Ar-etched and (b) pristine and $\mathrm{O}_{2}$-etched BP FETs at a $V_{\text {Ds }}$ bias of $50 \mathrm{mV}$. The channel length and width of the devices are $2 \mu \mathrm{m}$ and $3 \mu \mathrm{m}$, respectively. Optical micrographs of the devices pre- and post-etching are shown as insets. 


\section{Supplementary Information}

\section{Effects of plasma-treatment on the electrical and optoelectronic properties of layered black phosphorus}

Sruthi Kuriakose, Taimur Ahmed, Sivacarendran Balendhran, Gavin E. Collis, Vipul Bansal, Igor Aharonovich, Sharath Sriram, Madhu Bhaskaran and Sumeet Walia*

Ms. S. Kuriakose, Mr. T. Ahmed, Dr. S. Balendhran, Assoc. Prof. S. Sriram, Assoc. Prof. M. Bhaskaran, Dr. S. Walia

Functional Materials and Microsystems Research Group and Micro Nano Research Facility, RMIT University, Melbourne VIC 3001, Australia

*E-mail: sumeet.walia@rmit.edu.au

Dr. G.E. Collis

CSIRO Manufacturing, CSIRO, Bayview Avenue, Clayton 3168, Victoria, Australia

Prof. V. Bansal

Ian Potter NanoBioSensing Facility and NanoBiotechnology Research Laboratory, School of Science, RMIT University, Melbourne VIC 3001, Australia

Assoc. Prof. I. Aharonovich

School of Mathematical and Physical Sciences, University of Technology Sydney, Ultimo, NSW 2007, Australia 
S1: AFM images and thickness profile of the etched flakes.
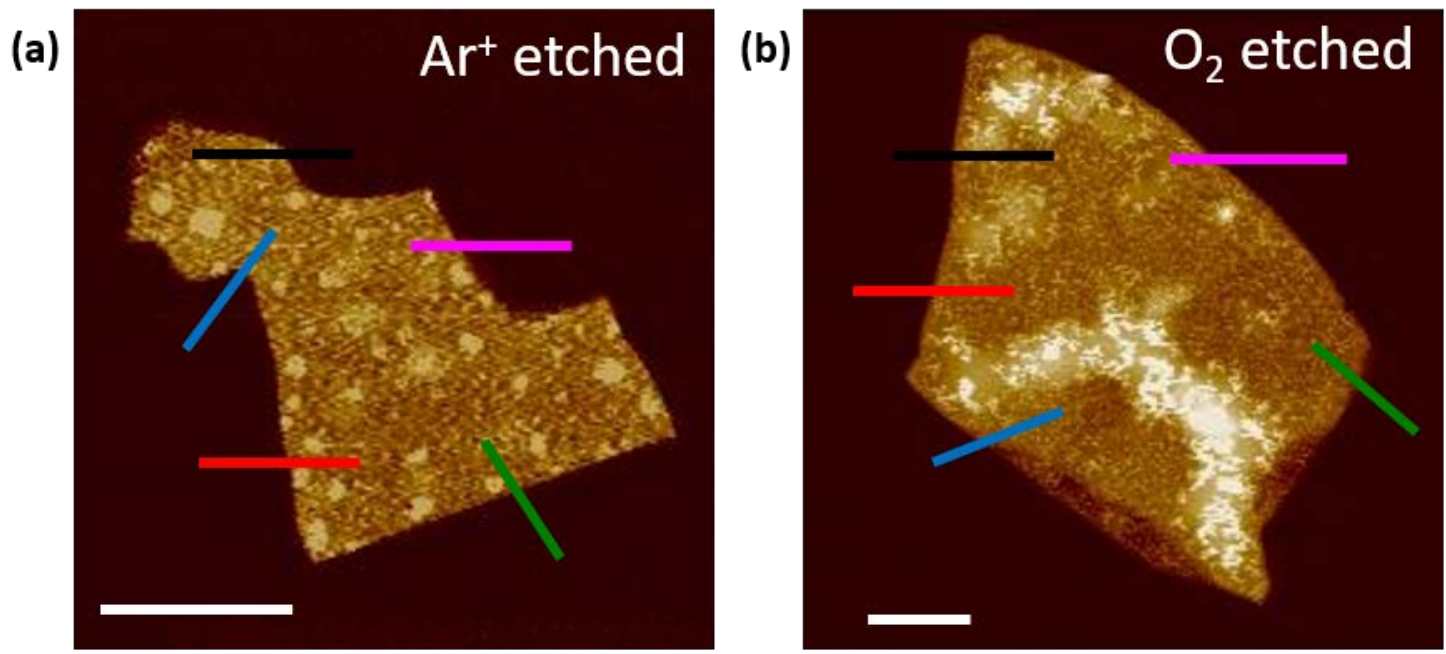

(c)

(d)
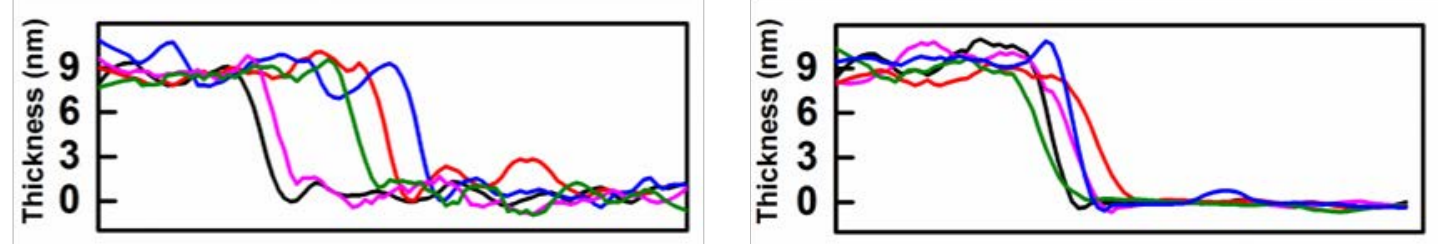

Figure S1: The AFM images of the flakes after plasma-assisted etching with $\mathrm{Ar}$ (a) and $\mathrm{O}_{2}$ plasma (b) respectively along with their marked areas of the thickness profiling. The scale bars of the image represent $2 \mu \mathrm{m}$. Thickness profiles of the $\mathrm{Ar}$ (c) $\mathrm{O}_{2}$ (d) etched flakes across the flake as indicated in (a) and (b). 

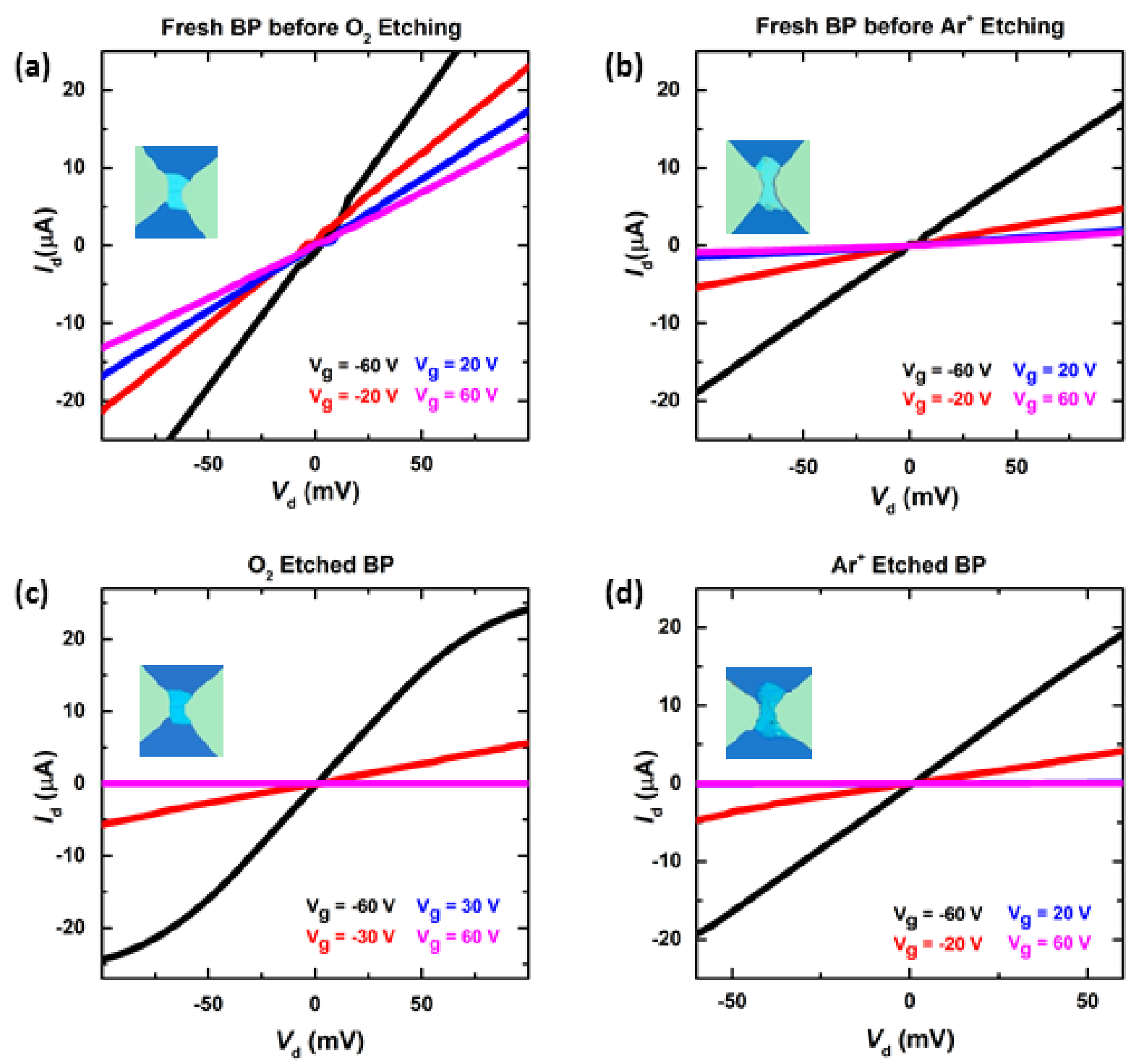

Figure S2: The $I-V$ characteristics of the devices with the as exfoliated BP (a) and (b). The $\mathrm{O}_{2}$-etched (c) and Ar-etched (d) are shown with their respective gate voltages. The inset shows the optical image of the device in $100 \times$ magnification.

The calculation of mobility was done using the equation[47]

$$
\mu=\frac{\Delta I_{\mathrm{ds}}}{\Delta V_{\mathrm{ds}}} \times \frac{L}{\text { W.C. } V_{\mathrm{ds}}}
$$

Where $\frac{\Delta I_{\mathrm{ds}}}{\Delta V_{\mathrm{ds}}}$ is the transconductance, $L=2 \mu \mathrm{m}$ is the length of the channel, $W=3 \mu \mathrm{m}$ is the width of the channel, $C=115 \mu \mathrm{Fm}^{-2}$ is the gate oxide capacitance, $\mathrm{V}_{D S}=50 \mathrm{mV}$ is the volatge across drain and source. The gate oxide capacitance was calculated using equation[47] $C=\frac{\varepsilon_{o} \times \varepsilon_{r}}{d}$, where $\varepsilon_{o}=8.854 \times 10^{-12} \mathrm{~F} / \mathrm{m}, \varepsilon_{r}=3.9$ for $\mathrm{SiO}_{2}$ and $\mathrm{d}$ is the thickness of the gate oxide, here $300 \mathrm{~nm}$. 